\title{
Determination of Inactive Powers in a Single-Phase AC Network
}

\author{
Nickolay I. Shchurov ${ }^{1}$, Sergey V. Myatezh ${ }^{1}$, Boris V. Malozyomov ${ }^{1, *}$, Alexander A. Shtang ${ }^{1}$, \\ Nikita V. Martyushev ${ }^{2} \mathbb{D}$, Roman V. Klyuev ${ }^{3}$ and Sergei I. Dedov ${ }^{1}$
}

1 Faculty of Mechatronics and Automation, Novosibirsk State Technical University, 20, Karla Marksa Ave., 630073 Novosibirsk, Russia; nischurov@mail.ru (N.I.S.); serg_y_7578@mail.ru (S.V.M.); shtang@corp.nstu.ru (A.A.S.); dedov_s.i@mail.ru (S.I.D.)

2 Department of Materials Science, Tomsk Polytechnic University, 30, Lenina Ave., 634050 Tomsk, Russia; martjushev@tpu.ru

3 Department of Low Temperature Engineering, Moscow Polytechnic University, 33, B. Semenovskaya Str., 107023 Moscow, Russia; kluev-roman@ramler.ru

* Correspondence: borisnovel@mail.ru

Citation: Shchurov, N.I.; Myatezh, S.V.; Malozyomov, B.V.; Shtang, A.A.; Martyushev, N.V.; Klyuev, R.V.; Dedov, S.I. Determination of Inactive Powers in a Single-Phase AC Network. Energies 2021, 14, 4814. https://doi.org/10.3390/en14164814

Academic Editor: Ahmed F. Zobaa

Received: 3 July 2021

Accepted: 4 August 2021

Published: 7 August 2021

Publisher's Note: MDPI stays neutral with regard to jurisdictional claims in published maps and institutional affiliations.

Copyright: (c) 2021 by the authors. Licensee MDPI, Basel, Switzerland. This article is an open access article distributed under the terms and conditions of the Creative Commons Attribution (CC BY) license (https:// creativecommons.org/licenses/by/ $4.0 /)$.

\begin{abstract}
Based on the development of the theory of reactive power and distortion power, starting with the works of Fryze and Budeanu, it has been found that the contradictions in the definition of the components of inactive powers are caused by errors in the introduced intermediate concepts and corresponding calculations when switching to nonlinear and non-sinusoidal AC circuits. The materials of the works of modern researchers and the numerical calculations carried out made it possible to trace the differences between reactive power and distortion power, to confirm the orthogonality properties of the active, reactive power, and distortion power components. The paper defines the conditions for achieving a power balance in an AC network with nonlinear loads, compiled and tested criteria leading to the absence of distortion power in a single-phase AC network. Using the time base of the projection of the generalized vectors in vector diagrams, it is shown that compliance with the criteria for the absence of distortion power does not determine the mutual similarity of the voltage curve with the current curve for a nonlinear load. It has been found that the well-known term "distortion power" has an unfortunate wording, since this power, although it characterizes the interaction of harmonics of currents and voltages with different ordinal numbers, is not determined by the visual similarity or the degree of distortion of the load current waveforms relative to the supply voltage curve.
\end{abstract}

Keywords: resource saving; energy efficiency; power quality capacitor bank; current harmonics; reactive power; hybrid power filter; active power filter

\section{Introduction}

At the initial stage of the power theory development, known since the beginning of the 20th century for sinusoidal AC networks, there were no contradictions in the formulations and calculated expressions [1,2]. Instead of instantaneous values of sinusoidal voltages $u(t)$ and currents $i(t)$, for the convenience of analysis and calculations in the well-known symbolic method, it is proposed to use their effective values $U$ and $I$. The product of these values $U \cdot I$ determines the total or apparent power $S=U \cdot I$. The reactive nature of the network $z$ elements explains the phase shift $\phi$ between voltage $u(t)=\sqrt{2} U_{1} \sin (\omega t)$ and current $i(t)=\sqrt{2} I_{1} \sin (\omega t+\phi)$. In this case, the apparent power $S$ consists not only of active power $P=U I \cos (\phi)$ capable of performing work, but also of ballast reactive power $Q=U I \sin (\phi)$. The components $P$ and $Q$ make up the power balance $S=\sqrt{P^{2}+Q^{2}}$, in which the proved orthogonality of $P$ with respect to $Q$ [1,2] is emphasized.

However, the appearance of loads with nonlinear current-voltage characteristics (CVC) led to a significant distortion of the network currents' curve shape and of supply 
voltages, which resulted in the formation of other inactive power components that do not perform useful work. It is obvious that the interaction of harmonics of non-sinusoidal voltage and current will create its own components of reactive power, etc. from each harmonic separately.

However, in this case, the excess of power $S$ over power $P$ can no longer be explained by the emergence of only reactive power $Q_{\Sigma}$ because of all harmonics. In the scientific literature $[3,4]$, this circumstance is explained by the appearance of another component of inactive power, which is known as the power of $D$ distortions. However, such view is not supported by all researchers and, to this day, it causes controversy about the correct definition of the combination of $Q_{\Sigma}$ and $D$, and their delineation taking into account higher harmonics.

For about 100 years, discussions have continued on the definition of the terminology for components $Q_{\Sigma}$ and $D$, their calculation, and the correct interpretation of their physical nature [4-7].

\section{Formulation of the Problem}

At present, AC electrical networks do not have strictly sinusoidal voltages, and most loads have sharply nonlinear I-V characteristics, which in turn causes non-sinusoidal currents to flow through all elements of electrical networks. Therefore, an important task is to study the processes of formation of individual power components due to the action of individual harmonics of voltages and currents and to illustrate their calculations using examples of simple loads in a single-phase alternating current network.

The practical value of the research results in this article is determined by its contribution to solving the problem of calculating inactive powers in the AC network. A clear understanding of the processes of formation of components of inactive powers will make it possible to clarify their formulations and definitions from the standpoint of physical interpretation and draw up appropriate expressions for the correct calculation of the values of reactive powers and distortion powers in a non-sinusoidal AC network.

This will allow one to correctly calculate each power component in the AC network, to create new, more advanced devices for analyzing and metering electrical energy, and to successfully solve urgent energy optimization problems of redistribution and compensation of inactive power.

In addition, the proven statements of the theory of power for single-phase alternating current networks can be subsequently applied to three-phase alternating current networks, where the occurrence of asymmetric modes significantly complicates the analysis if there is no clear representation of inactive and full power $[8,9]$.

\section{Aspects in the Development of Inactive Capacities Theories}

In the historical aspect, the works of many scientists are devoted to the definition of inactive powers in non-sinusoidal AC networks. The work of Budeanu [3] describes one of the first explanations of the mechanism of occurrence of the power imbalance, when the power is decomposed into orthogonal components $P$ and $Q$, and defining $Q_{\sum}$ as a set of reactive powers $Q_{i}$ caused by individual harmonics:

$$
Q=\sum Q_{i}=\sum U_{i} I_{i} \sin \left(\phi_{i}\right)
$$

where $i$ is the ordinal numbers of the harmonics of the effective values of voltages $U_{i}$ and currents $I_{i} ; \phi_{i}$ is the shear angles between them. It is in this theory that the power balance is achieved by introducing an additional component of inactive power, mutually orthogonal with respect to the already known components and in such a way that:

$$
S=\sqrt{P^{2}+Q^{2}+D^{2}}
$$

The value $D$ was presented as a measure of the mutual distortion of the curves $u(t)$ and $i(t)$. However, it has already been noted in [10] that, in AC networks with certain 
combinations of higher harmonics at the level of instantaneous powers, the interaction of the components $Q$ and $D$ can occur and in such a way that $D=0$ even in conditions of a distorted shape of the curve $i(t)$ relative to $u(t)$. Vice versa, $D \neq 0$ provided that the shape of the curve $i(t)$ is similar to the shape of the curve $u(t)$. This fact gave rise to doubts about the validity of the Budeanu theory and the search for other approaches to determining the inactive power that do not contradict the results of the experiments.

Such approaches were available in early works by Fryze $[2,10,11]$. In them, inactive power $Q_{q}$ is not divided into components $Q$ and $D$ or the like, but it is defined as a power imbalance:

$$
Q_{q}=\sqrt{S^{2}-P^{2}}=\sqrt{\frac{1}{T} \int_{0}^{T} u^{2}(t) d t \cdot \frac{1}{T} \int_{0}^{T} i^{2}(t) d t-\left(\frac{1}{T} \int_{0}^{T} u(t) i(t) d t\right)^{2}} .
$$

Since, by definition, $Q_{q}$ cannot do the job, the Fryze theory is built on dividing the instantaneous load currents into two components: active $i_{a}(t)$ and reactive $i_{r}(t)$ currents, in such a way that $i(t)=i_{a}(t)+i_{r}(t)$. Assuming that the currents' components $i_{a}(t)$ and $i_{r}(t)$ are mutually orthogonal, their respective effective values $I_{a}^{2}$ and $I_{r}^{2}$ will define the initial current as $I^{2}=I_{a}^{2}+I_{r}^{2}$.

Hence, there is no explicit physical interpretation for power $Q_{q}$ in the representation of the Fryze theory, as it has been done for sinusoidal circuits.

Subsequent works [12,13], in which the development of the theory of powers is based on frequency techniques, are devoted to the establishment of the relationship between the higher harmonics of voltage and current with the components of inactive powers. This defines reduced power, and, using the ratios of the frequencies of the fundamental and higher harmonics with ordinal numbers $k$, the following determination of inactive power $Q$ has been obtained:

$$
Q=\sum k \cdot Q_{k}=\sum k \cdot U_{k} I_{k} \sin \left(\phi_{k}\right)
$$

However, it, like many other similar calculated expressions in practice, had not always provided a power balance $[10,14]$ in an AC network with a non-sinusoidal shape $u(t)$ and $i(t)$.

In subsequent works [15], an attempt was made to further divide the current $i_{r}(t)$ into orthogonal components: inactive current and a residual component. The inactive current determines the parameter of the passive reactive element (inductance or capacitance). For the harmonics with ordinal numbers $n$, this approach provides an expression for determining $Q$ :

$$
Q=\sum \frac{1}{n} U_{n} I_{n} \sin \left(\phi_{n}\right) \sqrt{\sum U_{n}^{2} / \sum \frac{U_{n}^{2}}{n}}
$$

The value $Q$ in these works was obtained as a result of reducing the reactive nature of the network elements, and, from a practical point of view, it allows for determining the parameters of the compensation device for the inactive current component using passive elements. However, the origin and physical meaning of the residual component of the inactive current are not considered.

The component of current $i_{a}(t)$, according to the Fryze theory, was determined in subsequent works by Czarnecki $[7,16]$ in accordance with expression (1) and the development of the provisions of the CPT (Conservative Power Theory). The inactive part was analyzed from the point of view of frequency calculation methods according to the CPC (Currents Physical Components) theory that are also using the methodology of orthogonal decomposition [17]. In this representation, reactive power is defined as the result of a large number of orthogonal components of currents $I_{i}$ of different frequencies and is calculated as:

$$
Q_{Z}=U \sqrt{\sum I_{i}^{2}\left(\sin \left(\phi_{i}\right)\right)^{2}}
$$


The total power of a linear load supplied from an AC network with a non-sinusoidal $u(t)$, in accordance with this theory, is $S=\sqrt{P^{2}+D_{S}^{2}+Q_{Z}^{2}}$. The component $D_{S}$ has been named as the dissipated power generated by the current components depending on the frequency spectrum $u(t)$. Therefore, only the component $Q_{Z}$ can be compensated by means of passive elements [7]. If the load turns out to be nonlinear, then this theory allows one to single out another component of the current $i_{g}(t)$, the harmonics of which are not determined by the spectrum of the periodic function, but are independently generated by the nature of the load itself [16], forming the corresponding power component $D_{g}$. In this case, the total power in a single-phase network will be determined by the combination of already four components $S=\sqrt{P^{2}+D_{S}^{2}+Q_{Z^{2}}+D_{g}^{2}}$.

Thus, in studies using frequency, spectral, and similar calculation methods based on the concepts of effective values of voltages and currents, no unity was found in the quantitative determination of the components of inactive powers and their physical interpretation.

In order to exclude possible errors in determining the power components, made in the course of intermediate calculations based on instantaneous values $u(t)$ and $i(t)$, neither frequency nor spectral, but integral techniques were used in the works of individual researchers, which do not require finding the effective values of harmonics. Thus, in the methods [13,18], the nature of the load is determined through its I-V characteristic. For this purpose, the area of the closed figure of the I-V characteristic of the load in the axes $u(t)$ and $i(t)$ for the period $T=2 \pi$ has been determined by integration as a value [19] that is proportional to the power $Q$ :

$$
Q= \pm \frac{S_{I-V}}{2 \pi}= \pm \frac{\int_{t}^{t+T_{0}} u \frac{d i}{d t} d t}{2 \pi} .
$$

A similar approach is described in [13], where the value $Q$ was obtained in the course of the cyclic integration operation:

$$
Q= \pm \frac{1}{2 \pi} \oint i(u) d u \text { or } Q= \pm \frac{1}{2 \pi} \oint u(i) d u .
$$

It is easy to see that expressions (2)-(8) not only characterize the components of inactive capacities in different ways, but, in the case of calculations, they will lead to a significant discrepancy between the obtained results.

Therefore, in recent years, in the theory of inactive powers, the tendency of direct analysis of instantaneous values has dominated, which has become the basis of the theory of instantaneous powers. The advantage of the theory of instantaneous powers is the elimination of intermediate concepts and calculations, which, when applied to nonlinear and non-sinusoidal AC circuits, can lead to fundamental errors in definitions and calculations due to an incorrect interpretation of the physical nature of the emergence of individual power components.

The concept of considering the instantaneous values of voltages and currents, applied in the first works of Fryze [2,11], was later used in [20] as the FBD theory (Fryze, Buchholtz, Depenbrock), described in more detail in [21,22]. The theory of instantaneous powers was further developed in the concept of the pq-theory [23], which determines the instantaneous values of active $p(t)$ and reactive $q(t)$ power through combinations of the products of the values of projections $u(t)$ and $i(t)$ onto orthogonal axes. The consequence of the development of the theory of instantaneous powers was its generalization in the crossvector theory $[17,24]$, in which the value $p(t)$ is defined as the scalar product of vectors $\vec{u}(t)$ and $\vec{i}(t)$, and the value $q(t)$ is defined as their vector product.

It should be noted that the provisions of individual theories of inactive power are capable of solving a number of practical problems with some success, for example, analyzing the operating modes of electrical networks or designing technical means to compensate for 
reactive power [25]. However, until now, none of the presented theories have yielded an unambiguous physical interpretation or explanation of the physical meanings of all the components of inactive powers, their occurrence, and interaction for arbitrary shapes of curves $u(t)$ and $i(t)$.

This hinders the development of effective calculation methods and the adoption of relevant standards, in which, first of all, there would be a clear, relatively simple, and unambiguous definition of the process of occurrence of each power component for the subsequent calculation of the total inactive power in electrical networks in which nonsinusoidal voltages and currents operate. This is evidenced by the IEEE 1459-2010 Standard adopted at due time [26], in which the concept of inactive power is introduced, but the definition of power $Q$ is limited only by the first harmonic.

\section{Analysis of Inactive Power Components in Wave and Vector Diagrams}

If voltage and current harmonics, for example, the first harmonics $i=j=1$, with the same ordinal numbers interact in the AC network, then, from the point of view of the symbolic method [1], the current vector $\bar{I}_{1}$ can either lag behind or ahead of the supply voltage vector $\bar{U}_{1}$ by an angle $\phi$. Characterizing these values as $u(t)=U_{1 \mathrm{~m}} \sin (w t)$ and $i(t)=I_{1 m} \sin (w t-\phi)$, the instantaneous load power $p(t)=u(t) \cdot i(t)$ is conventionally divided into a constant component $p_{=}$and an alternating component $p_{\sim}$ :

$$
p(t)=U_{1 m} \sin (w t) \cdot I_{1 m} \sin (w t-\phi)=\frac{U_{1 m} I_{1 m}}{2}[\cos (\phi)-\cos (2 w t-\phi)]=p_{=}+p_{\sim} .
$$

The integral sum of the components $p_{=}$and $p_{\sim}$ for the period $T$ corresponding to the full reversal of the vectors $\bar{I}_{1}$ and $\bar{U}_{1}$ is capable of forming active power $P_{1}$, being determined by the argument $\phi_{1}$ as $P_{1}=\frac{1}{T} \int_{0}^{T} u_{1}(t) i_{1}(t) d t=\frac{U_{1 m} I_{1 m}}{2} \cos \left(\phi_{1}\right)$, or proceeding to the effective values $U_{1}=\frac{U_{1 m}}{\sqrt{2}}, I_{1}=\frac{I_{1 m}}{\sqrt{2}}$ :

$$
P_{1}=U_{1} I_{1} \cos \left(\phi_{1}\right) \text {. }
$$

In this connection, $\frac{1}{T} \int_{0}^{T} p_{=} d t=P, \frac{1}{T} \int_{0}^{T} p_{\sim} d t=0$. The appearance of an alternating value $p(t)$ in the combination of components $p_{=}$and $p_{\sim}$ over a period $T$ characterizes the action of exchange or reactive power $Q$ between the elements of the AC network, for example, a source and a load. As it follows from expression (9), the sign $p(t)$ will change at a frequency of $2 w t$ twice over the period $T$.

The quantity $Q_{1}$ can be traditionally expressed through the unbalance of total power $S_{1}=U_{1} I_{1}$ and active power $P_{1}$, using the well-known property of orthogonality of the components $Q$ and $P$ as in the case of $Q^{2}=S^{2}-P^{2}$, which gives the well-known expression $[1,3]$ :

$$
Q_{1}=\sqrt{U_{1}^{2} I_{1}^{2}\left(1-\cos ^{2}\left(\phi_{1}\right)\right)}=U_{1} I_{1} \sin \left(\phi_{1}\right) .
$$

As the vector $\bar{I}_{1}$ lags behind the vector $\bar{U}_{1}$, the component $p_{=}$decreases, and negative values $p(t)$ appear and grow, which indicates a decrease in the component $P_{1}$ and the appearance of a growing component $Q_{1}$.

If the harmonics of voltage $\bar{U}_{i}$ and current $\bar{I}_{\mathrm{j}}$, having different ordinal numbers $i \neq j$, interact in an $\mathrm{AC}$ network, then, due to the orthogonality property [27] of these harmonics with multiple frequencies $\mathrm{j} / i=n$ during the working period $T$, the work cannot be done, since, in this case, the power is:

$$
P_{i j}=\frac{1}{T} \int_{0}^{T} u_{i}(t) \cdot i_{j}(t) d t=0 .
$$


It makes no sense to further decompose such power into components $p_{=}$and $p_{\sim}$. However, as it follows from expression (3), the total power in this case will be a value $S_{i j}=U_{i} I_{j} \neq 0$ that is commonly called the distortion power $S_{i j}=D_{i j}$.

In this way, in an alternating current network, even with a sinusoidal voltage source, a load with a purely active character is capable of generating powers $Q_{i}$ and $D_{i j}$, if it has a nonlinear I-V characteristic.

It is common knowledge that, if an artificial delay occurs in the opening of an electronic key in a single-phase AC network supplying a purely active load, a spectrum of higher current harmonics will form [5-7]. In this spectrum, only the first harmonic of the current will create a component $Q_{1}$.

As an example, Figure 1 shows oscillograms and the spectral composition (Table 1) of the load current with $R=1$ p.u. in a network with a sinusoidal voltage source of $U=100 \mathrm{p} . \mathrm{u}$. and the frequency of $f=50 \mathrm{~Hz}$ when the delay in the opening of the electronic keys was about $70 \%$ or $0.007 \mathrm{~s}$.

In such network with a sinusoidal voltage of the power supply, a load with a purely active character causes a phase shift in the fundamental current harmonic of $-54.8^{\circ}$, as if the load electrical circuit contained an inductive element, the reactive power of which can be formally calculated by expression (11). However, it is, of course, erroneous to assume that such circuits contain reactive elements that exchange power with a power source. Moreover, in separate works $[15,17]$, it was noted that the concept of reactive power, which "characterizes the energy pumped from the source to the reactive elements of the receiver and then returned by these elements back to the source during the same period of oscillation, referred to this period", does not fully reflect the physical meaning.

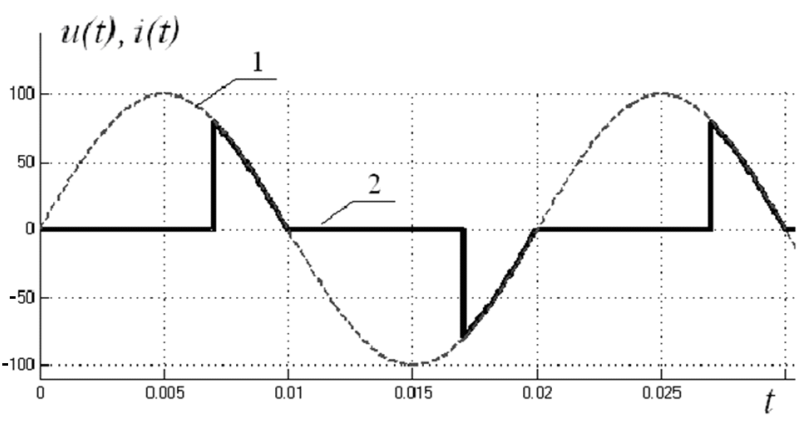

(a)

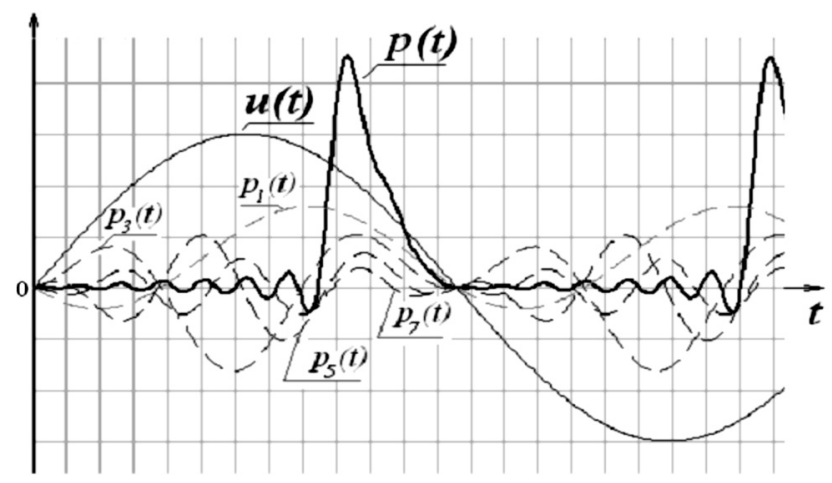

(b)

Figure 1. Wave diagrams: (a) oscillograms of the supply voltage (curve 1) and active load current (curve 2); (b) synthesis of harmonics of instantaneous load powers.

Table 1. Spectral composition of active load current.

\begin{tabular}{cccc}
\hline Harmonic No. & Harmonic Frequency $f_{\boldsymbol{i}}, \mathbf{( H z )}$ & Amplitude $\boldsymbol{I}_{\boldsymbol{m} i}, \mathbf{( A )}$ & Phase Shift, $\boldsymbol{\phi}_{\boldsymbol{i}}$ \\
\hline 1 & 50 & 24.46 & $-54.8^{\circ}$ \\
3 & 150 & 20.11 & $16.9^{\circ}$ \\
5 & 250 & 13.28 & $94.2^{\circ}$ \\
7 & 350 & 7.16 & $188.5^{\circ}$ \\
9 & 450 & 5.03 & $-51.6^{\circ}$ \\
11 & 550 & 5.02 & $53.8^{\circ}$ \\
15 & 650 & 4.12 & $150.8^{\circ}$ \\
\hline
\end{tabular}

If the action of each harmonic (Table 1) is analyzed separately in this example, then it is not too difficult to see in Figure 2a that the vector of the current third harmonic $\bar{I}_{3}$, built according to the data in Table 1 , is three times ahead of the voltage vector per one period $T$. 
The interaction of vectors $\bar{U}_{1}$ and $\bar{I}_{3}$ will create instantaneous power $p_{3}(t)$, the alternating pulsations of which (Figure 2b), due to the properties of expression (12), are distributed in such a way that the total area bounded by the curve $p_{3}(t)$ with positive values is equal to the total area bounded by this curve with negative values so that $P_{13}=0$. At the same time, the total power in this case is $S_{13}=U_{1} I_{3}=D_{13}=711.8 \mathrm{~V}$.A. In Figure 3 waveforms explaining the power generation for the fifth (Figure $3 a$ ) and seventh (Figure $3 b$ ) harmonics are shown. The remaining higher harmonics of current interacts with voltage $\bar{U}_{1}$ in a similar way; this is accompanied by the appearance of powers $P_{15}=0, D_{15}=664 \mathrm{~V} \cdot \mathrm{A}$, $P_{17}=0, D_{17}=358 \mathrm{~V} \cdot \mathrm{A}$.

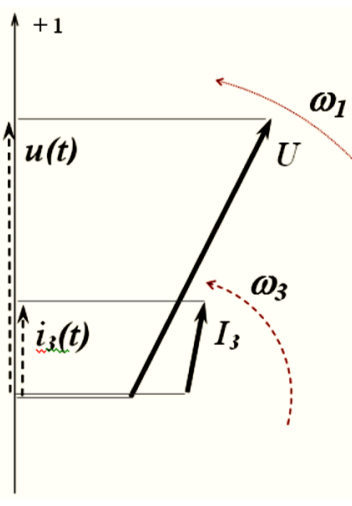

(a)

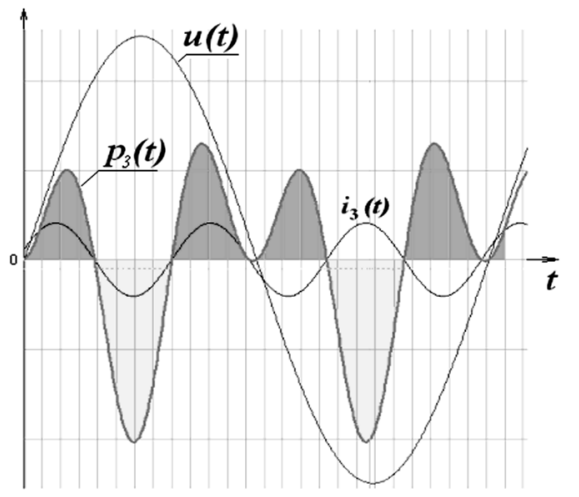

(b)

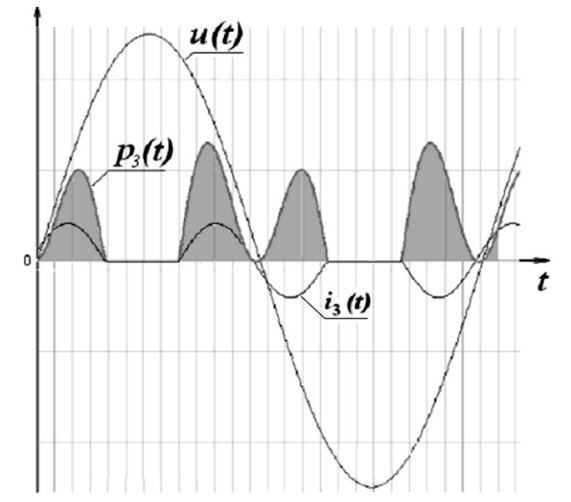

(c)

Figure 2. Vector and wave diagrams: (a) vector diagrams of the power supply voltage and the third harmonic of the load current; $(\mathbf{b}, \mathbf{c})$ wave diagrams of instantaneous voltages, currents, and powers.

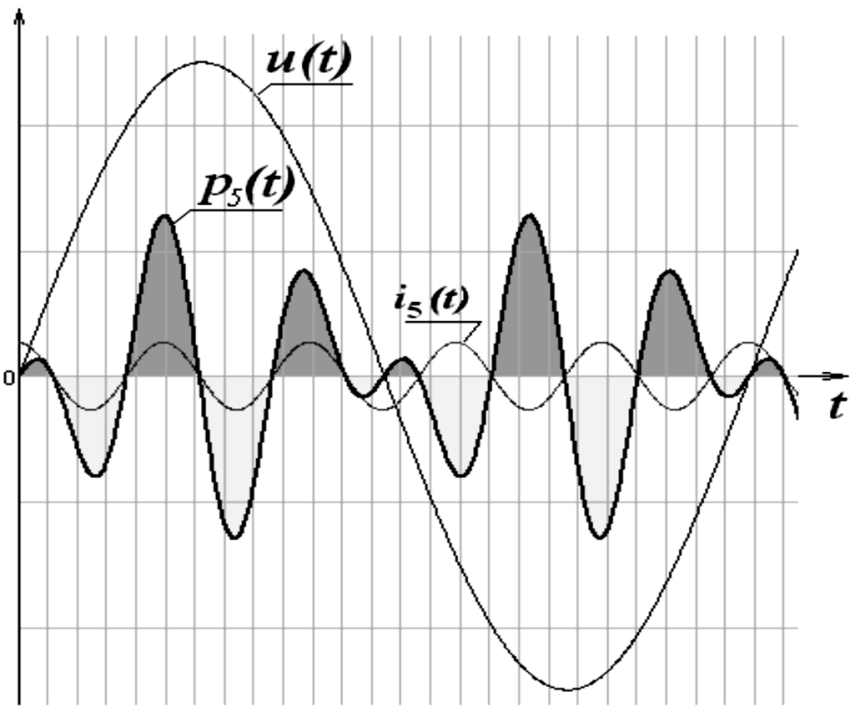

(a)

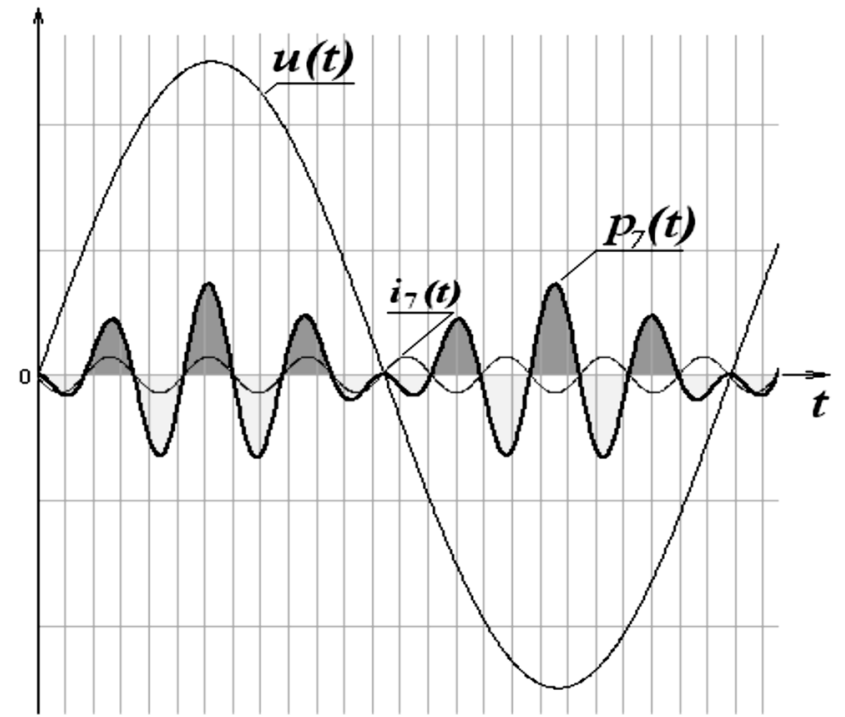

(b)

Figure 3. Wave diagrams of the supply voltage and higher harmonics of current and instantaneous powers: (a) fifth harmonic; (b) seventh harmonic.

Thus, the actions of the instantaneous values of the components of $p_{i}(t)$, taking into account all higher harmonics, are completely mutually neutralized in the time intervals when the electronic switch is open (Figure 1b). This example shows that the power $Q_{1}=U_{1} I_{1} \sin \left(\phi_{1}\right)$ from the first harmonic of the current is not the prerogative of the manifestation of the properties of reactive elements in the AC network, but it can be 
considered as an imbalance of instantaneous values $p_{i}(t)$ that have caused a shift in the components $p_{=}$and $p_{\sim}$.

In fact, power $Q_{1}$ interacts with power components $D_{i j}$ at the level of redistribution of instantaneous values $p(t)$, while the values of the components $Q_{1}$ and $D_{i j}$ remain unchanged [28]. This is due to the fact that one harmonic, characterizing a certain power component, as follows from the properties of the Fourier series [27], cannot be obtained or compensated for by a set of other harmonics. In this case, it is possible to correct the shape of the curve $p(t)$ of an arbitrary AC consumer by regulating the phase shifts of the quantities $p_{i}(t)$ created, for example, in accordance with expression (12) using an inactive power compensator with zero active power [29-31].

The set of values with orthogonal properties of harmonics is written as:

$$
D_{\Sigma}=\sqrt{\sum_{i=1}^{\infty} D_{i}^{2}}
$$

In this example, there is only one voltage harmonic $\bar{U}_{1}$; therefore, $D_{\Sigma}=\sqrt{\sum_{i=2}^{\infty} U_{1}^{2} I_{i}^{2}}$. According to Table 1 , this value is $D_{\Sigma}=\frac{100}{\sqrt{2}} \sqrt{\left(\frac{20.11}{\sqrt{2}}\right)^{2}+\left(\frac{13.28}{\sqrt{2}}\right)^{2}+\left(\frac{7.16}{\sqrt{2}}\right)^{2}+\left(\frac{5.03}{\sqrt{2}}\right)^{2} \ldots \approx}$ 1347.4 V·A.

Active and reactive powers calculated using Table 1 by expressions (10) and (11) have the following values:

$$
\begin{gathered}
P_{1}=U_{1} I_{1} \cos (\phi)=\frac{100}{\sqrt{2}} \cdot \frac{24.46}{\sqrt{2}} \cos (54.7) \approx 712 \mathrm{~W} \\
Q_{1}=U_{1} I_{1} \sin (\phi)=\frac{100}{\sqrt{2}} \cdot \frac{24.46}{\sqrt{2}} \sin (54.7) \approx 1005 \mathrm{~V} \cdot \mathrm{A} .
\end{gathered}
$$

This provides the power balance according to expression (2), since:

$$
S=U \cdot I=\frac{100}{\sqrt{2}} \sqrt{\left(\frac{24.46}{\sqrt{2}}\right)^{2}+\left(\frac{20.11}{\sqrt{2}}\right)^{2}+\left(\frac{13.28}{\sqrt{2}}\right)^{2}+\left(\frac{7.16}{\sqrt{2}}\right)^{2} \ldots} \approx 1825.46 \mathrm{~V} \cdot \mathrm{A} .
$$

This yields $S=\sqrt{P^{2}+Q^{2}+D^{2}}$ or $1825.46 \approx \sqrt{712^{2}+1005^{2}+1347.4^{2}}$.

This confirms the idea in the Budeanu theory [3] that the powers $P, Q$, and $D$ are mutually orthogonal, and the difference $\sqrt{S^{2}-P^{2}}$ determines the totality of inactive powers $Q^{2}+D^{2}$.

\section{Analysis of Budeanu Theory Criticisms}

Despite the fact that calculations similar to the above-mentioned example prove the consistency of the Budeanu's theory [3], a number of researchers, for example [4,9,10,32], pose a number of questions:

(1) Why, in practice, expression (2) is not true for all measured modes of operation of consumers in the alternating current network;

(2) Why distortion power $D$ can occur in conditions when the load current curve is similar to the supply voltage curve and vice versa; $D=0$ provided that the load current curve is clearly distorted relative to the supply voltage curve;

The answer to the first question is determined, first of all, by the reasons that violate the property of harmonics orthogonality (12). This is possible if the parameters of the higher harmonics of the current (amplitude, phase shift) are not stationary during the operating period $T$, which is quite typical of AC circuits containing modern semiconductor devices. This will lead to a violation of the equality of the areas bounded by the curves $p_{i}(t)$ for positive and negative values, as is shown in Figure 2b. In such cases, individual current harmonics with non-stationary parameters, interacting with the mains voltage $\bar{U}_{1}$, are able to do work because, for them, $P_{i} \neq 0$. 
For example, let us assume a simple condition that per one period:

$$
\begin{gathered}
i_{3}(t)=I_{3} \sin \left(3 w t+\phi_{3}\right), \text { if } i_{3}(t) \cdot u_{1}(t) \geq 0 \text { and } \\
i_{3}(t)=0, \text { if } i_{3}(t) \cdot u_{1}(t)<0,
\end{gathered}
$$

one can obtain a pulsating, but positive power in Figure 2c. It is not too difficult to see that, as a result of the accepted condition, a harmonic component $i_{1}(t)$ will appear in the spectral composition of the curve $i_{3}(t)$ shown in Figure 2c, the lagging character of which will create an additional $Q_{1}=31.8 \mathrm{~V}$. A component relatively $u_{1}(t)$.

As a result, the active power calculated based on the first and highest harmonics will be:

$$
P_{\Sigma}=\sum_{i=1}^{\infty} P_{i}=(712+417)=1129 \mathrm{~W} ;
$$

reactive power:

$$
Q_{\Sigma}=\sum_{i=1}^{\infty} Q_{i}=(1005+31.8)=1036.8 \mathrm{~V} \cdot \mathrm{A}
$$

distortion power:

$$
\begin{aligned}
& D_{\Sigma}=\sqrt{\sum_{i=2}^{\infty} D_{i}^{2}}=\sqrt{0+711.8^{2}+664^{2}+358^{2}+251.5^{2}+251^{2}+206^{2}+156^{2}}=1145.2 \mathrm{~V} \cdot \mathrm{A} ; \\
& S_{\Sigma}=\sqrt{\sum_{i=1}^{\infty} S_{i}^{2}}=\sqrt{1231.6^{2}+825^{2}+664^{2}+358^{2}+251.5^{2}+251^{2}+206^{2}+156^{2}}=1732.8 \mathrm{~V} \cdot \mathrm{A} .
\end{aligned}
$$

Violation of the power balance $\sqrt{P^{2}+Q^{2}+D^{2}} \neq S$ or $\sqrt{1129^{2}+1036.8^{2}+1145.2^{2}}=$ $1913.4>1732.8$ is just evidence of the incorrectness of calculating the effective values of the harmonics of currents or voltages under conditions of dynamic change in the parameters of the harmonic composition per one measurement period $T$, when the higher harmonics did not have constancy in amplitudes and phase shifts.

In this way, the power components $P_{i}, Q_{i}$, and $D_{i j}$ must be methodically correctly calculated based on the instantaneous power values $p(t)=u(t) \cdot i(t)$, which contain the most reliable information on the operating modes of the AC network.

To answer the second question, it is necessary to analyze the conditions for the appearance of $D \neq 0$-when the curves $i(t)$ and $u(t)$ are similar, and $D=0$, when the curve $i(t)$ is distorted relative to the curve $u(t)$.

The condition $D=0$ with the explicit presence of the spectral composition of the harmonics of the distorted curve $i(t)$ relatively $u(t)$ unambiguously indicates the compensation of the components in expression (13), which is possible in the case when each component $D_{i j}=0$.

Let, for example, the curves $u(t)$ and $i(t)$ form two harmonics of voltages $U_{m}, U_{n}$ and currents $I_{m}$ and $I_{n}$. In this case, the harmonics $m$ and $n$ form active powers according to expression (10):

$$
P_{m}=U_{m} I_{m} \cos \left(\phi_{m}\right) \text { and } P_{n}=U_{n} I_{n} \cos \left(\phi_{n}\right) .
$$

They form reactive powers according to expression (11):

$$
Q_{m}=U_{m} I_{m} \sin \left(\phi_{m}\right) i Q_{n}=U_{n} I_{n} \sin \left(\phi_{n}\right) .
$$

Proceeding from expression (2), the following can be obtained:

$$
D^{2}=S^{2}-\left(P^{2}+Q^{2}\right), S^{2}=(U \cdot I)^{2}=\left(U_{m}^{2}+U_{n}^{2}\right) \cdot\left(I_{m}^{2}+I_{n}^{2}\right) .
$$


Hence, the distortion power can be expressed as:

$$
D=\sqrt{U_{m} I_{n}\left(U_{m} I_{n}-2 U_{n} I_{m} \cos \phi_{m} \cdot \cos \phi_{n}\right)+U_{n} I_{m}\left(U_{n} I_{m}-2 U_{m} I_{n} \sin \phi_{m} \cdot \sin \phi_{n}\right)} .
$$

Therefore:

$$
D=0 \text { if } U_{m} I_{n}\left(U_{m} I_{n}-2 U_{n} I_{m} \cos \phi_{m} \cdot \cos \phi_{n}\right)=-U_{n} I_{m}\left(U_{n} I_{m}-2 U_{m} I_{n} \sin \phi_{m} \cdot \sin \phi_{n}\right)
$$

This is possible under the condition of simultaneous achievement of the identity in the ratios of rms values for all harmonics of voltages and currents:

$$
\frac{U_{m}}{U_{n}}=\frac{I_{m}}{I_{n}}
$$

and the equality of phase shifts in all harmonics of voltages and currents:

$$
\phi_{m}=\phi_{n}
$$

If the harmonics $U_{m}, U_{n}, I_{m}, I_{n}$ that correspond to conditions (15) and (16) can be represented in the form of rotating vectors on the complex plane, then the projections of the resulting voltage $\bar{U}=\bar{U}_{m}+\bar{U}_{n}$ and current vectors $\bar{I}=\bar{I}_{m}+\bar{I}_{n}$, unfolded in time, form (Figure $4 \mathrm{c}$ ) a distorted curve $u(t)$ with respect to the curve $i(t)$. At the same time, the hodographs $\bar{u}(t)$ and $\bar{i}(t)$ of the resulting vectors are similar and mutually shifted by $\pi / 2$ (Figure $4 \mathrm{a}$ ). Along with this, the curves $u(t)$ and $i(t)$ characterize the formation of purely reactive power $S=Q=Q_{m}+Q_{n}$ in the AC network.

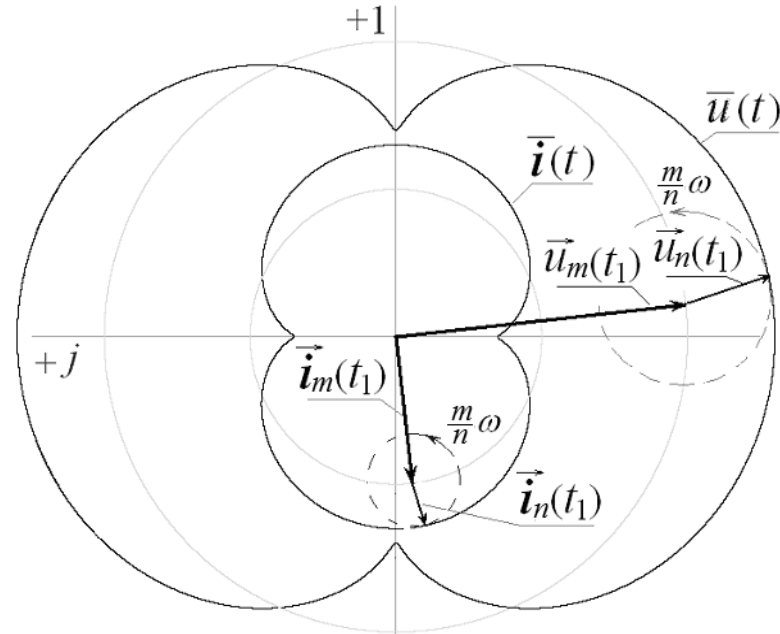

(a)

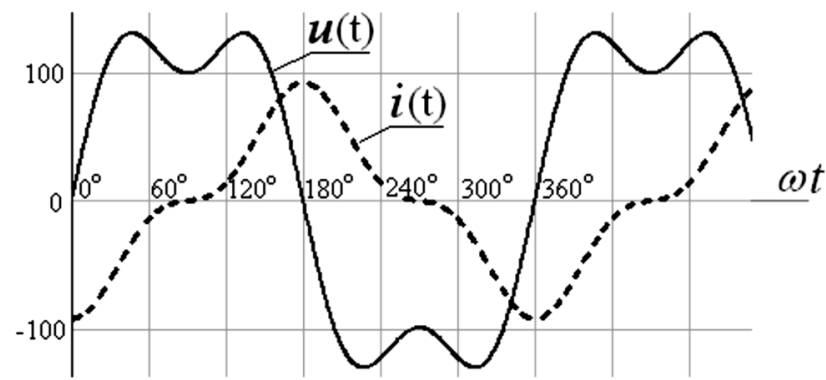

(c)

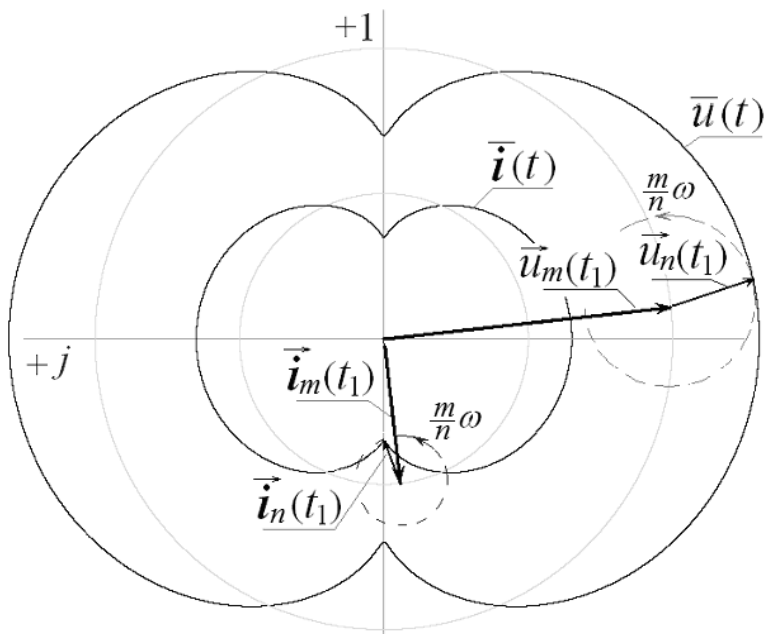

(b)

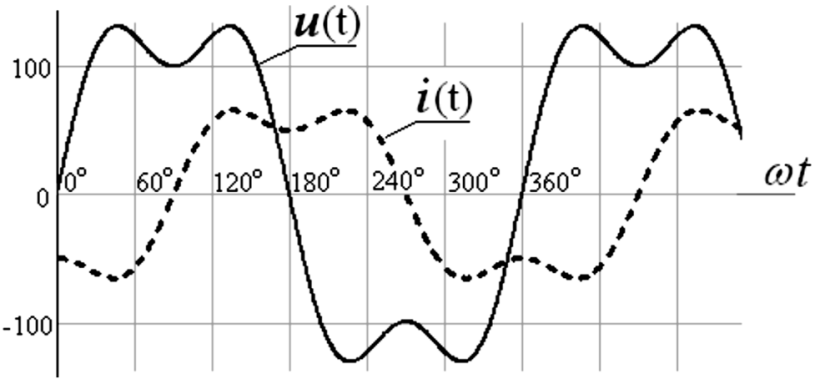

(d)

Figure 4. Diagrams of voltages and currents in a non-sinusoidal AC network containing harmonics $\mathrm{m}$ and $\mathrm{n}$ : $(\mathbf{a}, \mathbf{b})$ vector diagrams; (c,d) wave diagrams. 
An attempt to provide the similarity of the curves $u(t)$ and $i(t)$ with formal preservation of the identity in their harmonic composition (Figure $4 b, d$ ) leads to the fact that, in addition to reactive power $Q$, distortion power $D$ appears in the AC network, which is easily verified by numerical calculations using expressions (1)-(3), (10), (11), and (13).

In the case of a large number of harmonics in the AC network, expression (14) will take on a more cumbersome form [28,33]:

$$
D=\sqrt{\sum_{i}^{\infty} \sum_{\substack{j, i \neq j}}^{\infty} U_{i} I_{j}\left(U_{i} I_{j}-2 U_{j} I_{i} \cos \phi_{i} \cdot \cos \phi_{j}\right)+U_{j} I_{i}\left(U_{j} I_{i}-2 U_{i} I_{j} \sin \phi_{i} \cdot \sin \phi_{j}\right)} .
$$

However, this does not fundamentally affect the behavior of the powers $P, Q$, and $D$ in the AC network.

Thus, the power $D$, despite its unsuccessful name, does not characterize the degree of distortion of the curve $i(t)$ shape relative to $u(t)$, but it indicates the presence of such interactions of harmonics of currents and voltages with different ordinal numbers, which generate additional ballast powers that are not capable of performing useful work.

\section{Conclusions}

1. Based on the analysis of wave and vector diagrams, the work has shown that the inactive power of $N=\sqrt{S^{2}-P^{2}}$ in non-sinusoidal AC networks is not an indivisible component, but, unambiguously, it can be further divided into orthogonal components: the distortion power of the $D_{\Sigma}$ and the reactive power of the $Q_{\sum}$, that is, $N=\sqrt{Q_{\Sigma}^{2}+D_{\Sigma}^{2}}$, which can be unambiguously calculated analytically. Taking into account the behavior of the higher harmonics of currents and voltages, the power of $Q$ and $D$ can be generated in an arbitrary way by active elements with nonlinear volt-ampere characteristics.

2. It is shown that power $Q$ is formed during the interaction of voltage and current harmonics with the same ordinal numbers $Q_{\Sigma}=\sum Q_{i}$. In addition, power $D$ is formed during the interaction of voltage and current harmonics with different ordinal numbers, due to the orthogonal properties of which $D_{\Sigma}=\sqrt{\sum_{i=1}^{\infty} D_{i}^{2}}$. At the level of instantaneous values of $p(t)=u(t) \cdot i(t)$, the interaction of these powers makes it possible to correct the shape of the supply currents and voltages in the AC network. However, the average value of the powers $Q_{\Sigma}$ and $D_{\Sigma}$ should be zero by definition over the working period.

3. Analytical expressions are obtained for calculating all power components in a nonsinusoidal single-phase AC network, according to which, as an illustrative example, rotating generalized vectors of voltages and currents are constructed based on individual harmonics. The time base of the projections onto the axis of these generalized vectors yields wave diagrams of the instantaneous values of the $u(t)$ and $i(t)$ curves, which is of practical value for solving research problems. Thus, using generalized vectors, it has been found that compliance with the criteria for the identity of the ratios of the rms values for all harmonics of voltages and currents $\frac{U_{m}}{U_{n}}=\frac{I_{m}}{I_{n}}$ and the equality of phase shifts of all harmonics of voltages and currents $\phi_{m}=\phi_{n}$ to achieve $D=0$ in a non-sinusoidal network does not provide a visual similarity of curves $u(t)$ and $i(t)$.

4. It is shown that the visual similarity of curves $u(t)$ and $i(t)$ cannot serve as a criterion for the absence of distortion power $D$. Power $D$ actually does not characterize the degree of distortion of the shape of the curve $i(t)$ relative to $u(t)$, but indicates the presence of ballast interactions of harmonics of currents and voltages with different ordinal numbers. These, in addition to reactive power, generate additional corresponding ballast powers that are also not capable of performing useful work. 
5. The performed analysis and calculations confirm the validity of the foundations of the theory of Fryze and Budeanu regarding the concepts of active and inactive powers, orthogonality of components $P_{i}, Q, D$ and achieving a power balance in a network with nonlinear volt-ampere characteristics of the load, despite the absence of a strict definition of power. However, the dynamic change in the amplitudes and phase shifts of individual current and voltage harmonics characteristic of modern loads over the time period that is shorter than the operating period $T$ complicates the calculation of the actual values of the powers $Q$ and $D$. In addition, if the harmonic analysis is performed incorrectly, it can lead to calculation errors.

Author Contributions: Conceptualization, N.I.S. and B.V.M.; methodology, S.V.M.; software, N.V.M.; validation, N.I.S. and B.V.M.; formal analysis, R.V.K.; investigation, S.I.D.; resources, A.A.S.; data curation, A.A.S.; writing —original draft preparation, S.I.D.; writing-review and editing, N.V.M.; visualization, R.V.K. All authors have read and agreed to the published version of the manuscript.

Funding: This research was supported by the NSTU development program. This research was supported by the TPU development program.

Institutional Review Board Statement: Not applicable.

Informed Consent Statement: Informed consent was obtained from all subjects involved in the study.

Conflicts of Interest: The authors declare no conflict of interest.

\section{References}

1. Steinmetz, C.P. Theoretische Grundlagen der Starkstromtechnik; Autorisierte deutsche Ausgabe, übersetzt von J. Hefty, Ingenieur; Verlag von Vieweg \& Sohn: Braunschweig, Germany, 1903; p. 331.

2. Fryze, S. Reactive and apparent power in non-sinusoidal systems. Przeglad Elektrot 1931, 7, $193-203$.

3. Budeanu, C.I. Reactive and Fictitious Powers; Rumanian National Institute of Energy: Bucharest, Romania, 1927 ; No. 2.

4. Willems, J.L. Budeanu's reactive power and related concepts revisited. IEEE Trans. Instrum. Meas. 2011, 60, 1182-1186. [CrossRef]

5. Emanuel, A.E. Power Definitions and the Physical Mechanism of Power Flow; Wiley-IEEE Press: Hoboken, NJ, USA, 2010.

6. Filipski, P.S. AP-A misleading quantity in the non-sinusoidal power theories doomed to fail. Eur. Trans. Electr. Power 1993, 3, 21-26. [CrossRef]

7. Czarnecki, L.S. Minimisation of Reactive Power under Non-sinusoidal Conditions. IEEE Trans. Instrum. Meas. 1987, IM-36, 18-22. [CrossRef]

8. Ferrero, A.; Morando, A.P.; Ottoboni, R.; Superti-Furga, G. On the Meaning of the Park power components in three-phase systems under non-sinusoidal conditions. Eur. Trans. Electr. Power 1993, 3, 33-43. [CrossRef]

9. Emanuel, A.E. Powers in non-sinusoidal situations. A review of definitions and physical meaning. IEEE Trans. Power Deliv. 1990, 5, 1377-1384. [CrossRef]

10. Czarnecki, L.S. What is wrong with the Budeanu concept of reactive and distortion power and why it should be abandoned. IEEE Trans. Instrum. Meas. 1987, 3, 834-837. [CrossRef]

11. Fryze, S. Active, reactive and apparent powers in circuits with distorted voltages and currents. Elektrotechnische Z. 1932, 53, 596-599.

12. Shepherd, W.; Zakikhani, P. Suggested definition of reactive power for nonsinusoidal system. Proc. Inst. Electr. Eng. (IET) 1972, 119, 1361-1362. [CrossRef]

13. Majewski, O.A. Energy Indicators of Valve Converters; Energy: Moscow, Russia, 1978; p. 320.

14. Sharon, D. Power factor definitions and power transfer quality in nonsinusoidal situations. IEEE Trans. Instrum. Meas. 1996, 45, 728-733. [CrossRef]

15. Kusters, N.L.; Moore, W.J.M. On the definition of reactive power under non-sinusoidal conditions. IEEE Trans. Power Appl. Syst. 1980, PAS-99, 1845-1854. [CrossRef]

16. Czarnecki, L.S. Scattered and reactive current, voltage and power in circuits with non-sinusoidal waveforms and their compensation. IEEE Trans. Instrum. Meas. 1991, 40, 563-567. [CrossRef]

17. Sirotin, Y.A. Orthogonal components of three-phase current with asymmetric active-reactive load in a four-wire circuit. Electr. Eng. Electromechanics 2016, 3, 62-66. [CrossRef]

18. Sulaimanov, A.O.; Goldstein, E.I. Determination of inactive power and its components by arrays of instantaneous values of currents and voltages. Bull. Tomsk Polytech. Univ. 2005, 308, 44-47.

19. Illovici, M.A. Definition et measure de la puissance et de i'energie rectives. Bull. Soc. Franc. Electr. 1925, 5, 931-956.

20. Buchholtz, F. Das Begriffsystem Rechtleistung; Wirkleistung, totale Blindleistung; Selbstverlag: Munchen, Germany, 1950. (In German) 
21. Depenbrock, M. The FBD-Methods a generally applicable tool for analyzing power relations. IEEE Trans. Power Syst. 1993, 8 , 381-387. [CrossRef]

22. Depenbrock, M.; Marshall, D.A.; van Wyk, J.D. Formulating Requirements for a Universally Applicable Power Theory as Control Algorithm in Power Compensators. ETEP Eur. Trans. Elect. Power Eng. 1994, 4, 445-455. [CrossRef]

23. Akagi, H.; Nabae, A. The p-q Theory in Three-Phase System under Non-Sinusoidal Conditions. Eur. Trans. Electr. Power 1993, 3 , 27-31. [CrossRef]

24. Willems, J.L. A new interpretation of the Akagi-Nabae power components for non-sinusoidal three-phase situations. IEEE Trans. Instrum. Meas. 1992, 41, 523-527. [CrossRef]

25. Nabae, A.; Nakano, H.; Togasawa, S. An instantaneous distortion current compensator without any coordinate transformation. In Proceedings of the 1995 International Power Electronics Conference, Yokohama, Japan, 3-7 April 1995; Institute of Electrical Engineers of Japan: Chiyoda-ku, Japan; pp. 1651-16555.

26. IEEE Power and Energy Society. IEEE Standard Definition for the Measurement of Electric Power Quantities under Sinusoidal, Nonsinusoidal, Balanced or Unbalanced Conditions (IEEE std. 1459TM_2010); IEEE Power and Energy Society: New York, NY, USA, 2010

27. Ango, A. Mathematics for Electrical and Radio Engineers; tr. from French; The Science: Moscow, Russia, $1965 ;$ p. 779.

28. Myatezh, S.V.; Novolodskiy, M.V.; Kitova, E.T. Definition of distortion power in AC network and analysis of its reasons. IOP Conf. Ser. Mater. Sci. Eng. 2019, 560, 012113. [CrossRef]

29. Petrov, A.A.; Shchurov, N.I. Reactive power compensation and high-frequency distortions correction in Metro. Adv. Eng. Res. Actual Issues Mech. Eng. 2017, 27, 604-608.

30. Akagi, H.; Kanazawa, Y.; Nabae, A. Instantaneous reactive power compensator's comprising switching device without energy storage components. IEEE Trans. Ind. Appl. 1984, IA-20, 625-630. [CrossRef]

31. Raza, M. Active and reactive power control of hybrid offshore AC and DC grids. Automatika 2019, 60, 432-442. [CrossRef]

32. Shi, J.; Yang, W.; Xue, F.; Qiao, W.; Zhang, D. Reactive power optimization of an active distribution network including a solid state transformer using a moth swarm algorithm. J. Renew. Sustain. Energy 2019, 11, 035501. [CrossRef]

33. Abreu, T.; Soares, T.; Carvalho, L.; Morais, H.; Simão, T.; Louro, M. Reactive Power Management Considering Stochastic Optimization under the Portuguese Reactive Power Policy Applied to DER in Distribution Networks. Energies 2019, 12, 4028. [CrossRef] 